\title{
A conservative approach to replace missing teeth in the aesthetic zone with Maryland bridge - A case report
}

\author{
Manawar Ahmad $^{1 *}$, Hina Naim ${ }^{1}$, Abdullah Mohsin Adawi ${ }^{2}$, Abubakr Siddiq ${ }^{2}$, Hussam Mosa Zaud Mayidi ${ }^{2}$, Yahya Hefdollah Hakami ${ }^{2}$ and \\ Al Muthana Ali Alhazmi
}

${ }^{1}$ Assistant Professor, Department of Prosthetic Dental Sciences, College of Dentistry, Jazan University, Saudi Arabia

${ }^{2}$ BDS student, 6th year, College of Dentistry, Jazan University, Saudi Arabia

\begin{abstract}
Restoring the missing central incisors in the mandibular jaw is one of the most difficult esthetic challenges in dentistry. A space in the mandibular anterior region of the dental arch can produce a psychological impact on the young patient. Resin bonded bridges are highly effective treatment option in these situations to restore the oral function and aesthetics and result in high levels of patient satisfaction. Maryland bridges are the type of resin bonded bridge with certain advantages over conventional fixed dental prosthesis such as minimal removal of the tooth structure, minimal potential for pulpal trauma, supra gingival margin preparation and reduced time and cost. Provisional restorations are usually not required. Maryland bridges are cemented to the abutment tooth using electrolytic etching of the metal surface to retain the metal framework. After etching of the metal, the bond is stronger between the tooth surface and the prosthesis. The bridge retention has been enhanced by the development of resin cements which bond chemically to both the tooth surface and the metal alloy. However, there are certain limitations of resin bonded prosthesis such as short clinical crowns, long edentulous spans, restored or damaged abutments, para-functional habits, deep bite and compromised enamel hyperplasia. This case report mentions the advantages, disadvantages, indications, contraindications and a simplified technique to restore the missing mandibular central incisors in a young adult patient with fabrication of Maryland bridge.
\end{abstract}

\section{Introduction}

There are various treatment options available for the replacement of the missing mandibular anterior incisors such as implant, removable partial denture and fixed partial denture. Removable partial denture may cause the bone resorption and flattening of the interdental papillae in long term use however it can be used as interim prosthesis for initial esthetics. Conventional bridge requires adequate amount of tooth preparation of all the surfaces of the abutment tooth which may lead to the pulpal trauma and hypersensitivity in young adult patients. Due to the presence of large pulp chambers and unavailability of sufficient enamel, a more conservative and less invasive resin bonded prosthesis may be an alternative treatment alternative for such conditions to replace the missing tooth as well as preserve the remaining alveolar ridge and soft tissue.

Resin bonded or resin retained bridges (RBBs/RRBs) are a type of fixed dental prostheses that need a minimum amount of tooth preparation. They are bonded directly to the tooth structure with the help of the resin cement provided the preparation is restricted to the enamel surface only. These restorations primarily depend on the resin cement for its retention. Based on the mode of retention of the prosthesis and resin there are various forms have been developed such as mechanical retention, micro mechanical retention, macroscopic mechanical retention and chemical retention. These restorations were first described in the 1973 when the natural extracted tooth of the patient was cemented directly to the etched enamel surface with composite resin for a limited time period to provide esthetics. Then Rochette Bridge was designed by Rochette for periodontal splinting of the mandibular anterior teeth [1]. However, due to the bulky framework and limited adhesion to the enamel surface its use was restricted [2]. Later Moon and Hudgins developed 'Virginia bridge' at Virginia Commonwealth University in 1984. Lost salt technique was utilized to provide the macroscopic mechanical retention to the framework and the tooth surface. In spite of adequate retention of Virginia bridge, this framework was bulky. The 'Maryland Bridge' was developed at the University of Maryland. The bridge retention has been enhanced by the development of resin cements which bond chemically to both the tooth surface and the etched metal alloy. It provides micro mechanical retention. Thompson and Livaditis in 1983 developed the technique of electrolytic etching of $\mathrm{Ni}-\mathrm{Cr}$ and $\mathrm{Co}-\mathrm{Cr}$ alloy. However, the Maryland bridges are alloys specific. It is used only for non-precious alloys because precious alloys cannot be etched to provide micromechanical retention.

\section{Case report}

A 19 years old male patient reported with the chief complaint of missing anterior teeth in the mandibular anterior region and need for aesthetic restoration of the same with fixed dental prosthesis. $\mathrm{He}$ was wearing mandibular anterior removable partial denture from last 10 months. The patient gave the history of extraction due to

Correspondence to: Manawar Ahmad, Assistant Professor, Department of Prosthetic Dental Sciences, College of Dentistry, Jazan University, Saudi Arabia, E-mail: ahmad955mls@gmail.com

Key words: resin- bonded fixed partial denture, maryland bridge, fixed dental prosthesis, resin cement

Received: April 10, 2017; Accepted: April 24, 2017; Published: April 27, 2017 
trauma of mandibular central incisors one year back. His expectations were reasonable and his psychological profile was good. Intra oral examination revealed missing mandibular central incisors with a slight buccal defect in the gingiva in the anterior region of tooth \#31 and \#41 due to pressure exerted from wearing the removable partial denture from last 10 months (Figure 1). Replacement of the missing teeth with implant needed bone augmentation procedure to allow for optimal bone integration and stability of the implants. The patient did not agree to the proposed surgical treatment plan. Also on clinical and radiographic examination, the teeth demonstrated gingival margins much coronal to the cement-enamel junctions, and large pulp chambers. A conservative and minimally invasive adhesive bridge was planned to restore the missing mandibular central incisors. Diagnostic wax up was performed to achieve the final outcome of the fixed restoration (Figure 2).

Minimal tooth preparation of the abutments (\#32 and \#42) was performed following the standard technique on the lingual surfaces only. Care was taken to ensure that the preparations were not extended beyond the linguo-proximal line angles on the abutments. Lingual preparation ended $1 \mathrm{~mm}$ from the incisal edge and a light chamfer finish line was prepared $1 \mathrm{~mm}$ supragingivally. Parallel retentive grooves were made in each preparation on the surface facing the edentulous space.

Impression procedures were carried out with addition silicone (Express XT, 3M ESPE, Germany) and sent to the laboratory. A metal framework with 'wings' extending onto the preparations was fabricated with non-precious alloy (Figure 3). Metal try in of the frame work showed minimum interferences. Shade selection was done using Vita 3-D Master shade guide. The trial fitting of the prosthesis was done and then esthetics mastication and speech were evaluated (Figure 4). The laboratory technician was instructed to keep the metal wings of the prosthesis off the incisal third to prevent darkening of the tooth because of the inhibition of light transmission. The fitting surfaces of the 'wings' were sandblasted with alumina 250 microns to create micromechanical retentive surfaces for the cement. The restoration was cemented in place using universal self-etch resin cement (Rely X U100, $3 \mathrm{M}$ ESPE, Germany) (Figures 5 and 6). The occlusion was verified in centric and eccentric mandibular positions and it was make sure that

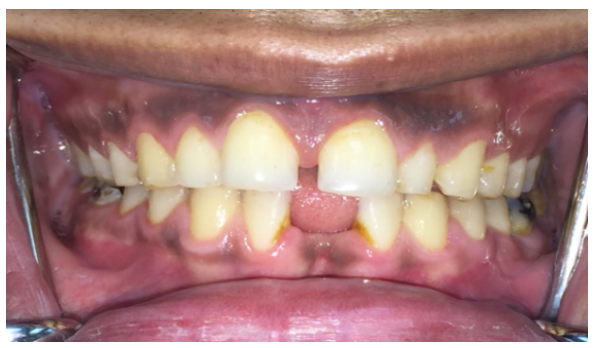

Figure 1. Intra oral view with missing mandibular central incisors.

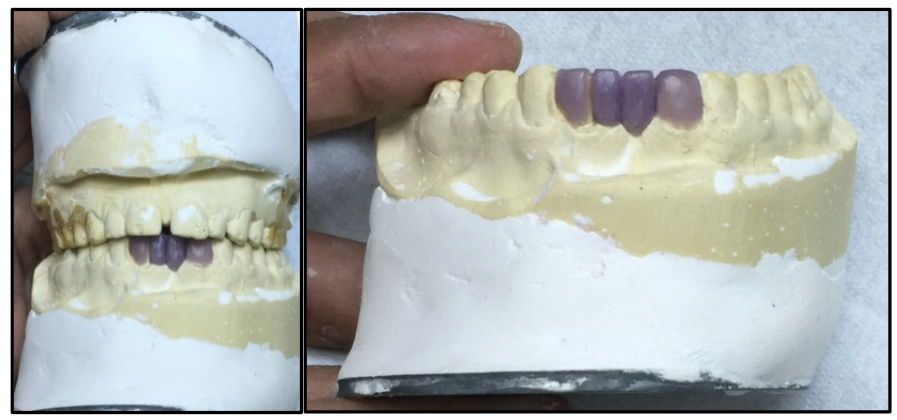

Figure 2. Diagnostic wax-up to evaluate the final restoration.

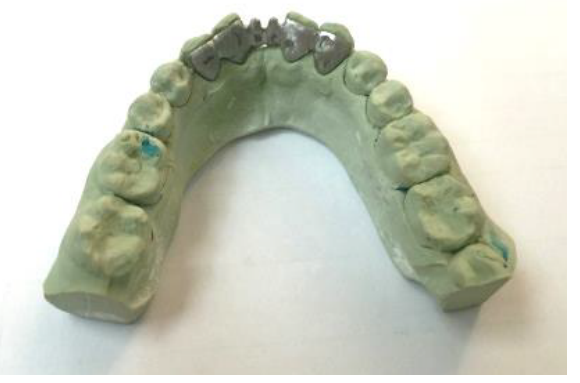

Figure 3. Fabrication of Maryland bridge metal frame work on the working cast

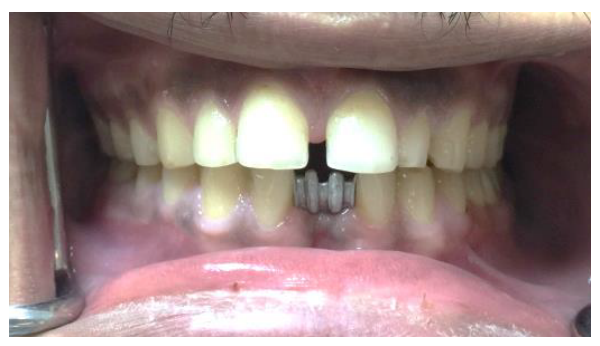

Figure 4. Intra oral view of metal try - in of Maryland bridge.

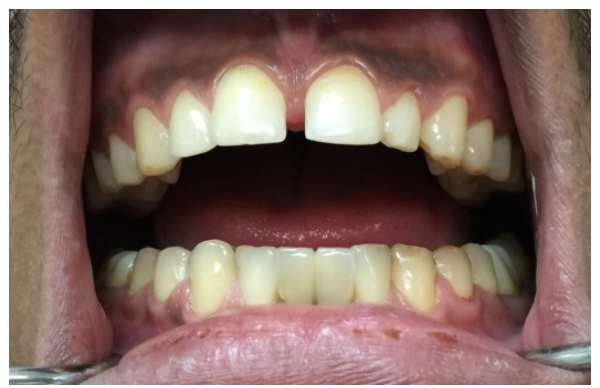

Figure 5. Intra oral view after cementation of Maryland bridge.

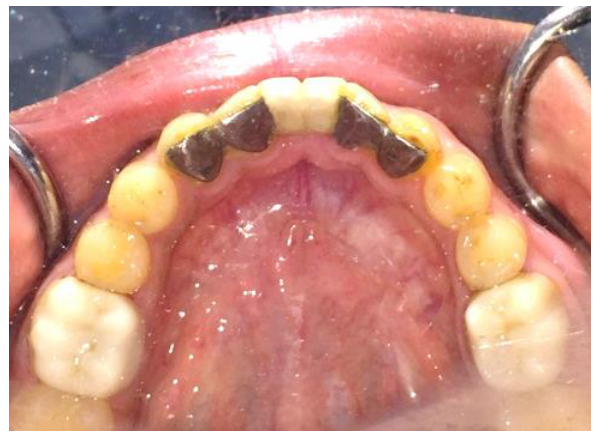

Figure 6. Lingual surface of Maryland bridge after cementation.

there was no interferences. Post cementation instructions were given and patient was followed up at regular intervals (Figures 7 and 8).

\section{Discussion}

Replacement of missing teeth with conventional fixed partial denture usually involves the conventional tooth preparation of all the surfaces of the adjacent teeth as abutments. Large pulp chambers in the abutments, expected transition in the position of the gingiva and age of the patient were factors that precluded the use of conventional fixed prostheses in this case. While these resin bonded restorations 


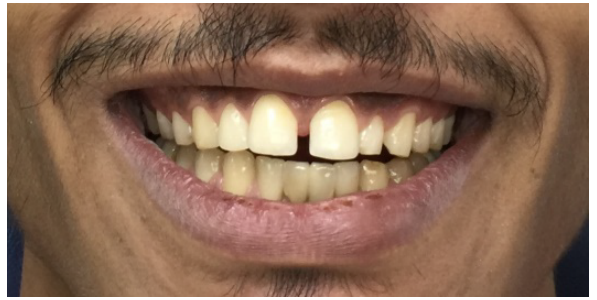

Figure 7. Post-operative smile of the patient.
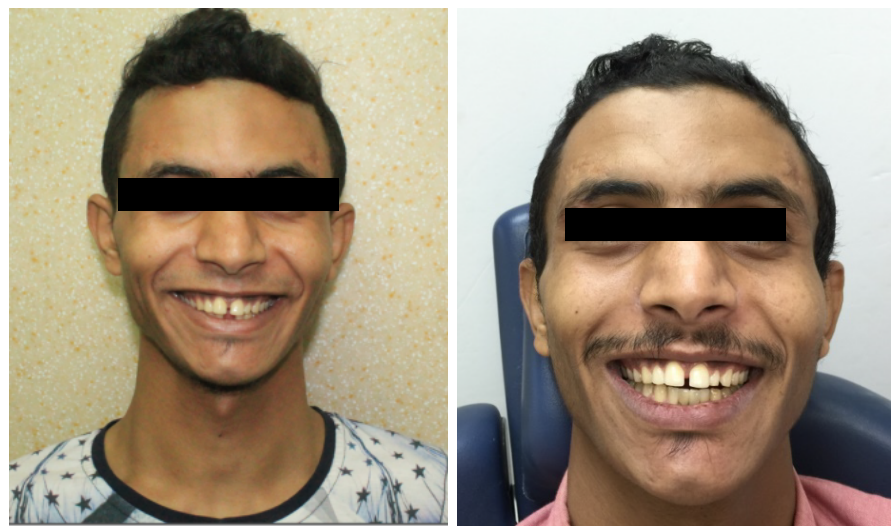

Figure 8. Extra oral view before and after cementation of Maryland bridge.

have compromised retention and corresponding life spans, newer selfetch adhesive systems help to ensure that such restorations are retained for reasonably long periods of time [3-6]. The new self-etch universal resin cement systems are valuable tools in ensuring longevity of such restorations that allow them to be in service for the intended period. The three most common complications associated with resin-bonded prosthesis is debonding (21\%), tooth discoloration (18\%) and caries $(7 \%)[7,8]$.

Although a $74 \%$ success rate at 4 years is considered satisfactory for adhesive restorations, the uneventful 1-year follow up in this case created much optimism regarding its tenure in service, at the end of which definitive prosthodontic treatment may be rendered [9-11].

\section{Conclusion}

Resin bonded bridges can be highly effective in replacing missing teeth, restoring oral function and aesthetics and result in high levels of patient satisfaction. Given thorough patient assessment and the use of careful clinical techniques, the resin bonded bridge should be considered more frequently as the restoration of choice for short spans.

\section{References}

1. Howe DF, Denehy GE (1977) Anterior fixed partial dentures utilizing the acid-etch technique and a cast metal framework. J Prosthet Dent 37: 28-31. [Crossref]

2. St George G, Hemmings K, Patel K (2002) Resin-retained bridges re-visited. Part 1 History and indications. Prim Dent Care 9: 87-91. [Crossref]

3. Stolpa JB (1975) An adhesive technique for small anterior fixed partial dentures. $J$ Prosthet Dent 34: 513-519. [Crossref]

4. Shilingburg HT, Hobo S (1997) Fundamentals of fixed prosthodontics, $3^{\text {rd }}$ edition, Quintessence Publishing Co Inc, IL, USA.

5. Jenkins CB (1985) The bond strength of new adhesive recommended for resin-bonded bridges. J Dent Res 64: 664.

6. Shilingburg HT, Hobo S (1997) Fundamentals of fixed prosthodontics, $3^{\text {rd }}$ edition, Quintessence Publishing Co Inc, IL, USA.

7. Pratyusha P, Jyoti S, Kaul RB, Sethi N (2011) Maryland bridge: An intrim prosthesis for tooth replacement in adolescents. Int J Clin Pediatr Dent 4: 135-138. [Crossref]

8. Goodacre CJ, Bernal G, Rungcharassaeng K, YK Kan J (2003) Clinical complications in fixed prosthodontics. J Prosthet Dent 90: 31-41. [Crossref]

9. Mukai M, Fukui H, Hasegawa J (1995) Relationship between sandblasting and composite resin-alloy bond strength by a silica coating. J Prosthet Dent 74: 151-155. [Crossref]

10. Creugers NH, Van 't Hof MA (1991) An analysis of clinical studies on resin-bonded bridges. J Dent Res 70: 146-149. [Crossref]

11. Jordan RE, Suzuki M (1978) Temporary fixed partial dentures fabricated by means of acid-etch resin technique: A report of 86 cases followed up for three years. J Am Dent Assoc 96: 994-1001. [Crossref]

Copyright: (C2017 Ahmad M. This is an open-access article distributed under the terms of the Creative Commons Attribution License, which permits unrestricted use, distribution, and reproduction in any medium, provided the original author and source are credited. 